Meta

Journal des traducteurs

Translators' Journal

\title{
La démarche de l'expert-traducteur face à un problème de reformulation
}

\section{Antin Fougner Rydning}

Volume 53, numéro 4, décembre 2008

URI : https://id.erudit.org/iderudit/019645ar

DOI : https://doi.org/10.7202/019645ar

Aller au sommaire du numéro

Éditeur(s)

Les Presses de l'Université de Montréal

ISSN

0026-0452 (imprimé)

1492-1421 (numérique)

Découvrir la revue

Citer cet article

Rydning, A. F. (2008). La démarche de l'expert-traducteur face à un problème de reformulation. Meta, 53(4), 748-764. https://doi.org/10.7202/019645ar

\section{Résumé de l'article}

Dans quels cas et pour quelles raisons l'expert-traducteur ressent-il un blocage dans la phase de reformulation ? Comment s'y prend-il pour débloquer la situation ? Une analyse des données in vivo de trois professionnelles norvégiennes de la traduction aux prises avec trois énoncés extraits d'un texte original français permet de montrer que la visualisation du sens joue un rôle essentiel dans la démarche cognitive de déblocage. 


\title{
La démarche de l'expert-traducteur face à un problème de reformulation
}

\author{
ANTIN FOUGNER RYDNING \\ Université d'Oslo, Oslo, Norvège \\ a.f.rydning@ilos.uio.no
}

\begin{abstract}
RÉSUMÉ
Dans quels cas et pour quelles raisons l'expert-traducteur ressent-il un blocage dans la phase de reformulation? Comment s'y prend-il pour débloquer la situation? Une analyse des données in vivo de trois professionnelles norvégiennes de la traduction aux prises avec trois énoncés extraits d'un texte original français permet de montrer que la visualisation du sens joue un rôle essentiel dans la démarche cognitive de déblocage.
\end{abstract}

\begin{abstract}
When and why does the professional translator experience problems when rendering the sense of utterances in his translation? How does he go about solving a reformulation problem? An analysis of on-line process data from three experienced Norwegian professional translators grappling with three utterances in a French original text shows that visualisation plays a key role in problem-solving on the level of reformulation.
\end{abstract}

\section{MOTS-CLÉS/KEYWORDS}

Analyse in vivo de données processuelles, visualisation du sens, concepts métaphoriques et métonymiques, processus complexe de résolution de problèmes de reformulation

\section{La traduction: un processus complexe de résolution de problèmes}

La traduction est par définition une activité intellectuelle complexe de résolution de problèmes. Même si le traducteur ne se heurte pas toujours à des problèmes - certains passages du texte à traduire ne demandant aucun effort apparent, ils peuvent en conséquence être traduits de manière automatique -, il arrive que des passages soient ressentis comme problématiques et nécessitent une réflexion poussée. C’est précisément la traduction réfléchie qui a retenu mon attention, et dont il sera question ici.

Dans le cadre de cet article, je propose de cerner de plus près la démarche du traducteur professionnel face à un problème de reformulation. Je tiens en particulier à observer dans quels cas et pour quelles raisons l'expert-traducteur ressent un blocage au moment de la reformulation de ce qu'il a compris, et comment il s'y prend pour débloquer la situation. Me basant sur les données processuelles de trois expertstraducteurs attestant chacune plus de 15 ans d'expérience active de la traduction professionnelle, je souhaite montrer que pour se sortir d'un blocage, le traducteur n'effectue pas une comparaison interlinguistique, mais adjoint des compléments notionnels au sémantisme du texte original (Lederer 1994: 52).

Pour pouvoir suivre les zigzags de la pensée réfléchie susceptible de déclencher les mécanismes cognitifs qui conduisent à un déclic, je prends appui sur les données processuelles in vivo de ces trois experts-traducteurs norvégiens aux prises avec leur traduction en norvégien d'un texte français. L'accent est mis sur trois énoncés qui 
ont donné lieu à un blocage. Le blocage se reconnaît d'une part aux pauses excédant 10 secondes enregistrées dans les données Translog des sujets traduisants - durée assimilable à un effort cognitif soutenu (Schilperoord 1996: 18; Jakobsen 2000: 167; Jensen 2000: 101; Rydning 2005: 102) - et d'autre part aux commentaires recueillis dans leur protocole de verbalisation.

\section{Méthodes d'observation in vivo de l'activité traduisante}

À défaut de pouvoir accéder directement à l'activité mentale déployée par le sujet traduisant, force est de recourir à des méthodes d'observation indirectes de la façon dont il s'acquitte de sa tâche. Partant du principe que la traduction se prête particulièrement bien à l'observation, puisque le traducteur se livre à une activité mentale poussée - laquelle peut être décelée par son protocole de verbalisation et/ou par sa façon de se mouvoir dans son texte, j'ai combiné deux méthodes d'observation in vivo des indices liés à son activité cognitive. Ces deux méthodes génèrent de riches données prises sur le vif pendant l'opération traduisante, qui lorsqu'elles sont étudiées en complémentarité, permettent d'obtenir des informations précieuses sur le cheminement cognitif du traducteur.

(i) Il s'agit d'une part des protocoles de verbalisation, mieux connus sous l'appellation de think-aloud protocols, couramment abrégés: TAPs, qui est une méthode d'enregistrement des réflexions du sujet traduisant aux prises avec son texte. Celui-ci est prié de verbaliser toutes les pensées qui lui passent par la tête au fur et à mesure qu'il exécute sa tâche. Ses réflexions enregistrées sur vidéo sont ensuite transcrites en protocoles de verbalisation. L'enregistrement vidéo permet d'observer un certain nombre d'activités cognitives, telles la lecture, l'écriture, la réflexion, la relecture, les corrections, la consultation de dictionnaires, la révision ainsi que ses états d'âme se manifestant aussi bien sous forme de données non verbales que de données auditives (Rydning 2004: 863), révélatrices d'efforts cognitifs. Importée de la psychologie cognitive (Ericsson et Simon 1980), la méthode TAPs est d'abord appliquée à la traduction pédagogique au milieu des années 1980 (Gerloff 1986; Krings 1986; Lörscher 1986), puis à la traduction professionnelle vers la fin des années 1980 (Jääskeläinen 1987; Séguinot 1989; Tirkkonen-Condit 1989) jusqu'à nos jours (Ericsson and Simon 1993; Jääskeläinen 2000; Tirkkonen-Condit 2002; Rydning 2002, 2005).

(ii) Il s'agit d'autre part de Translog - un logiciel développé par Jakobsen et Schou (Jakobsen 1999), qui permet d'enregistrer toutes les activités d'écriture du sujet, correspondant aux touches du clavier de l'ordinateur sur lesquelles a appuyé le traducteur, aux mouvements de la souris reliée à son ordinateur ainsi qu'aux pauses entre les activités d'écriture. L'avantage de Translog est qu'il permet d'observer ce que fait le traducteur sans pour autant le gêner dans l'accomplissement de sa tâche. Bien qu'il ait été prévenu que ses activités d'écriture et ses pauses seront enregistrées par le logiciel, l'enregistrement se fait à son insu. Par ailleurs, aucune compétence préalable n'est requise pour manier le programme Translog, qui se présente comme un simple programme de traitement de texte. Le texte original s'affiche dans la partie supérieure de l'écran, alors que la partie inférieure est réservée à la traduction. Les données informatisées sont stockées dans un fichier qu'il est possible de faire apparaître sur l'écran en deux versions : play-back cinématique et graphique. Dans le cadre 
du présent article, c'est la version graphique qui sera mise à profit. Celle-ci permet de visualiser non seulement les lettres correspondant aux touches sur lesquelles a appuyé le traducteur, mais aussi les lettres correspondant aux touches du clavier autres que celles qui renvoient aux lettres de l'alphabet ainsi qu'aux mouvements de la souris.

Les deux méthodes de collection des données processuelles in vivo se combinent parfaitement. Bien que le logiciel Translog ne soit pas équipé d'une fonction de synchronisation électronique permettant d'aligner les données Translog aux données TAPs, la synchronisation manuelle permet au chercheur de suivre les activités d'écriture en parallèle avec les commentaires verbaux, ce qui facilite l'interprétation qualitative des données.

Aux termes de l'analyse de la démarche cognitive de l'expert-traducteur, j'ai retenu les trois énoncés ci-dessous (en italique). L'énoncé 1) est la retombée linguistique d'une métaphore conceptuelle vue sous l'aspect de la personnification, c'est-àdire d'une extension de la métaphore ontologique qui consiste à percevoir une idée ou un objet comme une personne. L'énoncé 2) relève d'une métonymie, à savoir d'une relation qui implique une substitution que l'on peut conceptuellement représenter comme X REPRÉSENTE Y. L'énoncé 3) relève d'une "métaphtonymie», c'est-à-dire d'une métaphore fondée sur une métonymie:

1) Le retour des bonnes manières.

2) Mais elles restent aussi, plus sournoisement, un mode de tri social.

3) S'il est désormais bien vu de laisser au placard cravate et costume trois pièces le vendredi $[\ldots]$.

Ces trois énoncés figurent en caractères gras dans le texte ci-dessous extrait de l'Express, Société, du 4-11 janvier 2000:

\section{Le retour des bonnes manières}

On les croyait désuètes, balayées par l'ouragan post-soixante-huitard, promises à une mort imminente par la recrudescence des incivilités. On les imaginait figées, tout juste bonnes à illustrer les manuels d'éducation pour jeunes filles de bonne famille. Erreur. Liftées, épurées, les bonnes manières sont à nouveau plébiscitées par les Français. Les bonnes manières sont un signe d'intégration. Mais elles restent aussi, plus sournoisement, un mode de tri social. Raison de plus pour maîtriser les codes. Les entreprises exigent aujourd'hui de leurs employés qu'ils sachent communiquer. Le «BSAM» (bonjour-sourire-au revoir-merci) est enseigné partout. Conseillère en image personnelle, Hélène Choumiloff veille au grain. Ses «élèves» sont des cadres de sexe masculin de plus de 40 ans. Leur objectif: éviter les gaffes au cours des dîners d'affaires. «Beaucoup de mes clients se sont faits à la force du poignet. C'est l'ascension sociale qui crée la gêne», constate la conseillère. Pendant les exercices, dans la rue ou au restaurant, elle traque les failles. L'apprentissage peut durer trois mois. Les élèves sont très motivés. S'il est désormais bien vu de laisser au placard cravate et costume trois pièces le vendredi, nul ne tolère, désormais, les infractions au code de bonne conduite. (Laurence Albert)

Reproduisons, pour commencer, la traduction de chacune des trois expertstraducteurs accompagnée de ma re-traduction française, laquelle se veut aussi proche que possible de la version norvégienne, tout en respectant la syntaxe du français. 
Anne:

1) Tilbake til de gamle gode manérene. (Retour aux bonnes vieilles manières.)

2) De er også, på underfundig vis, en måte å foreta en sosial klassifisering på. (Elles sont aussi, de manière sournoise, une façon d'effectuer un classement social.)

3) Selv om det nå er god tone å la slips og dress bli hengende i skapet på fredagen [...]. (Même s'il est de bon ton aujourd'hui de laisser cravate et costume dans la penderie le vendredi $[\ldots]$.)

\section{Brigitte:}

1) Gode manerer er i vinden som aldri før. (Les bonnes manières sont plus que jamais dans le vent.)

2) Men de er fortsatt, om enn på en mer fordekt måte, også en metode for sosial sortering. (Mais elles sont toujours, bien que de façon plus dissimulée, une méthode de tri social.)

3) Selv om det er alment akseptert å la slips og dress henge i skapet på fredagene [...]. (Même s'il est communément accepté de laisser cravate et costume dans la penderie les vendredis [...].)

\section{Christine:}

1) Høflighet på mote igjen i Frankrike. (La politesse à la mode de nouveau en France.) 2) [...] men de er også, på en mer fordekt måte, et tegn på ens sosial posisjon. ([...] mais elles sont aussi, d'une façon plus dissimulée, un signe de la position sociale de chacun.)

3) Selv om det nå er akseptert at man henger fra seg dressen på fredagen [...]. (Même s'il est aujourd'hui accepté de ranger son costume dans la penderie le vendredi [...].)

\section{Les mécanismes de la métaphorisation, métonymisation et «métaphtonymisation" conceptuelles}

Pour comprendre comment la métaphore, la métonymie et la métaphtonymie conceptuelles jouent un rôle primordial dans l'élaboration du sens d'un énoncé, présentons brièvement ces trois concepts à la lumière de la théorie conceptuelle des métaphores et des métonymies, dites CTMM: the Cognitive Theory of Metaphor and Metonymy. Cette théorie pose que le sens des mots est encyclopédique. La connaissance encyclopédique est organisée en domaines d'expérience.

\subsection{Le mécanisme de la métaphorisation conceptuelle}

On pourrait se demander ce qu'il y a de métaphorique dans l'expression 1) qui constitue le titre du texte: Le retour des bonnes manières. Il convient à cet égard de se référer à la conception cognitiviste de Lakoff et Johnson (1980, 1984), Lakoff et Turner (1989), ainsi que Gibbs (1994) selon laquelle notre système conceptuel ordinaire, qui nous permet de penser et d'agir, est de nature fondamentalement métaphorique. La métaphore est l'un des instruments les plus puissants et les plus persuasifs de conceptualisation, elle est à la base des mécanismes de compréhension. Les métaphores ne sont donc pas perçues comme une simple déviation par rapport à ce qui est normal dans le langage. En fait, il n'y a rien de plus naturel que la métaphore - et les expressions métaphoriques fourmillent dans la langue. Elles sont plutôt la règle que l'exception. Elles prennent leur source dans l'expérience de l'être humain ainsi que dans la 
culture à laquelle appartient celui-ci. La compréhension de concepts abstraits passe par la compréhension de concepts plus proches de notre expérience physique du monde. Le mécanisme cognitif sur lequel se fonde cette compréhension est appelé «métaphorisation conceptuelle».

Une projection est établie entre deux domaines conceptuels. Une partie de la structure d'un concept peut donc être comprise métaphoriquement grâce à la possibilité d'établir une relation d'identité avec la structure d'un autre domaine. Cette relation d'identité, dite aussi projection domaniale, est essentielle pour comprendre le propre de la métaphore conceptuelle. Prenons les expressions métaphoriques usuelles suivantes puisées chez Lakoff et Johnson (1980: 49) en guise d'illustration:

She cast her spell over me.

The magic is gone.

I was spellbound.

She had me hypnotized.

He has me in a trance.

I was entranced by him.

I'm charmed by her.

She is bewitching.

Par voie de projection domaniale, chacune de ces expressions métaphoriques lie la structure de la magie à celle de l'amour. Les mots: cast her spell, magic, spellbound, hypnotized, in a trance, entranced, charmed et bewitching renvoient tous à un état magique. La métaphore conceptuelle sous-jacente est celle de L'AMOUR EST MAGIQUE. Du fait que la pensée métaphorique est réfléchie dans le langage, il convient de distinguer entre:

- le concept métaphorique qui est une image abstraite, une façon de structurer nos perceptions, représenté en lettres majuscules.

- l'expression métaphorique qui est la retombée linguistique de notre façon de concevoir l'image abstraite, représentée en lettres minuscules.

Les métaphores dites ontologiques nous permettent de voir les événements, les activités, les idées, les émotions comme des entités ou substances (Lakoff et Johnson 1980 : 25). Dès que nous sommes en mesure d'identifier nos expériences comme des entités, nous sommes à même d'y renvoyer, de les catégoriser, de les grouper, de les quantifier - bref de raisonner. La métaphore ontologique la plus caractéristique est celle de la personnification. C'est une métaphore par laquelle nous comprenons des concepts abstraits comme s'ils étaient des personnes. Voici la définition de la personnification proposée par Lakoff et Johnson (1980: 33):

Perhaps the most obvious ontological metaphors are those were the physical object is further specified as being a person. This allows us to comprehend a wide variety of experiences with nonhuman entities in terms of human motivations, characteristics, and activities. Here are some examples:

This theory explained to me the behaviour of chickens raised in factories.

This fact argues against the standard theories.

Life has cheated me.

Inflation is eating up our profits.

His religion tells him that he cannot drink French wines.

The Michelson-Morley experiment gave birth to a new physical theory.

Cancer finally caught up with him. 
Dans chacun des exemples ci-dessus, nous attribuons un comportement humain à une réalité non humaine. La raison d'être de la personnification est son pouvoir explicatif. Elle nous permet de comprendre des notions abstraites comme s'il s'agissait d'êtres humains. Voici ce que disent Lakoff et Turner au sujet de notre capacité de puiser dans notre for intérieur pour prêter aux objets et aux notions abstraites des caractéristiques humaines, voire des sentiments:

As human beings, we best understand other things in our own terms. Personification permits us to use our knowledge about ourselves to maximal effect, to use insights about ourselves to help comprehend such things as forces of nature, common events, abstract concepts, and inanimate objects. (Lakoff and Turner 1989: 72)

C'est justement ce type particulier de métaphore qui est à l'œuvre dans le titre du texte: Le retour des bonnes manières. Les commentaires de Christine sur un passage du texte dans lequel figure une autre expression métaphorique inédite portant elle aussi sur les bonnes manières, qu'elle relie cependant au titre, sont particulièrement révélateurs de cette inclination naturelle à attribuer des caractéristiques humaines à une notion abstraite:

1) Liftées, épurées, les bonnes manières sont à nouveau plébiscitées par les Français.

Commentaires TAPS de Christine (traduits du norvégien)

Les bonnes manières, vêtues plus simplement, s'apprêtent à faire leur rentrée et la bienvenue leur est souhaitée. [...] Peut-être que je pourrais dire «sont bien accueillies en France"; non, ça ne marche pas avec "vêtues plus simplement», ce qui m'obligerait à tout renverser. Allons: s'apprêtent à revenir vêtues plus simplement et sont accueillies [...]. Sont accueillies, sont reçues (ouvre les bras) à bras ouverts. [...] J'opte pour cette solution, qui est une espèce de personnification: des vêtements plus simples. Sont reçues à bras ouverts par les Français.

Christine confère aux bonnes manières les propriétés de personnes: celles-ci sont "vêtues plus simplement", "s'apprêtent à faire leur rentrée» et «sont accueillies, sont reçues à bras ouverts». Son commentaire sur l'accueil chaleureux réservé aux bonnes manières est ponctué par le geste d'ouvrir les bras. Christine est d'ailleurs parfaitement consciente de son recours à la personnification: "J'opte pour cette solution, qui est une espèce de personnification.»

Les bonnes manières peuvent conceptuellement être représentées comme si elles étaient des personnes dotées de la capacité de se mouvoir à travers le temps et l'espace: LES BONNES MANIÈRES SONT DES PERSONNES.

\subsection{Le mécanisme de la métonymisation conceptuelle}

La métonymie a été traitée comme le parent pauvre de la métaphore dans la littérature cognitiviste, bien qu'elle soit aujourd'hui considérée comme primordiale par la linguistique cognitive (Barcelona 2003; Taylor 2003). Panther et Radden (1999) sont d'avis que la métonymie est une entité encore plus importante dans la constitution du sens que la métaphore:

Eighteen years after Lakoff and Johnson's (1980) seminal work on the role of metaphor in conceptualization, which sparked a vast amount of research in cognitive linguistics, it has become increasingly apparent that metonymy is a cognitive phenomenon that may be even more fundamental than metaphor. (Panther and Radden 1999: 1) 
Les métonymies sont tout aussi courantes que les métaphores dans le langage quotidien. Leur compréhension se fait pratiquement à notre insu et relève de notre capacité de déduction usuelle. Comme l'a fait remarquer Gibbs (1994), les mécanismes cognitifs d'inférence mis en jeu pour comprendre un énoncé métonymique font partie de notre système conceptuel habituel:

Although metonymy has traditionally been viewed as a special rhetorical device in poetry and literature, it is a ubiquitous part of how we think of people, places, events, and things. Our conceptual ability to use one well-understood aspect of some domain to stand for the domain as a whole, or to use the mention of the whole domain to refer to one salient subpart, motivates our speaking so frequently in metonymic terms. Listeners are readily able to understand conventional and novel metonymic expressions precisely because the inferences needed to interpret such language are a common mental operation in our everyday conceptual system. (1994:358)

La métonymie correspond au procédé de désignation par lequel une relation d'identité entre deux sous-domaines est établie au sein d'un même domaine conceptuel. La métonymie figure aussi bien dans des idiomes, comme «l'Hexagone», qui désigne la France; des expressions figées comme, par exemple, "aspirer à la couronne», qui signifie qui aspire à devenir le souverain d'un pays; que dans des expressions inédites comme "the ulcer in room 201 " (Panther et Thornburg 2003: 282) où le terme "ulcer» renvoie au malade qui souffre d'un ulcère, ou encore: «l'électricien tricolore», qui sert à désigner l'EDF (Électricité de France).

À la différence de la métaphore, la projection conceptuelle est effectuée au sein d'un seul et même domaine cognitif. Un élément du schéma peut activer la totalité du schéma. Empruntons un exemple à Barcelona pour illustrer ce principe:

2) To keep my hand in I practice the piano on a regular basis (Barcelona 2000:37).

En prenant comme point de départ la métonymie conceptuelle conventionnelle BODY PART FOR AN ATTRIBUTE CONNECTED WITH ITS TYPICAL FUNCTION (Barcelona 2000: 37), le domaine source de la MAIN active l'un des sous-domaines cibles: L'ADRESSE MANUELLE. Cette activation nous fait comprendre l'idée centrale de la «dextérité au piano».

La métonymie conceptuelle fournit des schémas d'inférence naturelle (Thornburg et Panther 1997) qui mènent au raisonnement pragmatique nécessaire pour construire aussi bien la partie explicite que la partie implicite du sens. Panther et Thornburg $(2003$; 2004) soulignent que dans la relation d'identité établie entre un domaine source, dit véhicule, et un domaine cible, c'est surtout le domaine cible qui est mis en relief:

Metonymy highlights the target concept, but the source concept is in general still recoverable. (Panther et Thornburg 2003: 283)

[...] we argue that in a prototypical metonymy the target meaning is conceptually more prominent, i.e. more in focus of attention, than the source meaning. (Panther et Thornburg 2004)

Pour éviter de confondre la stratégie de conceptualisation qu'est la métonymie avec la celle de la métaphore, Croft (2003: 187) suggère de réserver le terme de projection domaniale: "domain mapping» à la métaphore, puisqu'une relation d'identité (similarité ou contraste) est établie entre deux domaines d'expérience différents, et d'utiliser 
celui d'actualisation domaniale: «domain highlighting» pour la métonymie, où il est question de projection intra-domaniale, dite aussi contiguïté, c'est-à-dire de juxtaposition entre deux sous-domaines d'un même domaine conceptuel.

Dans le deuxième énoncé du texte original: «Mais elles restent aussi, plus sournoisement, un mode de tri social», le domaine source MODE DE TRI SOCIAL active le sous-domaine cible FAÇON DE CLASSIFIER LES INDIVIDUS SUR UNE ÉCHELLE SOCIALE. C'est ce mécanisme de métonymisation qui nous met en mesure de saisir l'idée centrale: les individus sont catégorisés en fonction de certains critères, dont la maîtrise des bonnes manières.

\subsection{Le mécanisme de la «métaphtonymisation" conceptuelle}

La métaphore et la métonymie sont souvent confondues du fait que toutes deux établissent une relation d'identité entre deux entités conceptuelles. Prenons l'exemple de Barcelona (2003: 240) en guise d'illustration:

3) He fell in the war

L'énoncé 3) ci-dessus est polysémique, car il peut être compris aussi bien dans un sens métaphorique que métonymique. Si c'est l'image du soldat tué au champ de bataille que l'émetteur a en tête, nous avons affaire à une métonymie, où la partie «fell» (= est tombé) renvoie à «died» (= est mort), que nous traduirions en français par: «Il est mort au champ d'honneur.» Si c'est l'image du soldat décédé pendant la guerre que l'émetteur visualise, nous avons affaire à une métaphore, que nous traduirions en français par: "Il est mort pendant la guerre.»

D’autre part, la confusion fréquente entre la stratégie de la métonymisation et celle de la métaphorisation est due à leur interaction. Souvent, une métaphore est issue d'une métonymie ou se fonde sur elle. Dans d'autres cas, la métonymie se superpose à la métaphore. Au lieu de les considérer comme deux phénomènes cognitifs différents - distinction au demeurant souvent difficile à établir -, Goossens propose de les amalgamer, et a créé, à cet effet, le néologisme "métaphtonymie» (2003: 350).

Le troisième énoncé dans le texte original: «S'il est désormais bien vu de laisser au placard cravate et costume trois pièces le vendredi» est un exemple d'une métaphtonymie. L'expression métonymique «cravate et costume trois pièces » désigne une tenue vestimentaire formelle et interagit (i) avec le syntagme «le vendredi», lequel renvoie métonymiquement à la fin de semaine et métaphoriquement au concept du temps de repos, et (ii) avec le syntagme «laisser au placard», lequel renvoie métaphoriquement à ne pas porter. Le sens contextuel de l'énoncé peut être paraphrasé en ces termes: "Une tenue vestimentaire décontractée au travail en fin de semaine est recommandée.»

\section{Les données processuelles des trois experts-traducteurs}

Appuyons-nous maintenant sur les données processuelles des trois experts-traducteurs afin de pouvoir cerner de plus près leur démarche de déblocage face à un problème de reformulation d'une expression métaphorique et/ou métonymique. Pour des contraintes d'espace, seules les données processuelles portant sur un énoncé par 
expert-traducteur seront étudiées. Ainsi seront présentées les données processuelles de Christine relatives à la traduction du titre dans l'énoncé 1), d'Anne relatives à la traduction de l'énoncé 2) et de Brigitte relatives à la traduction de l'énoncé 3).

\subsection{Les données processuelles de Christine relatives à l'énoncé (1): Le retour des bonnes manières où chacun des astérisques qui n'est pas mis entre crochets correspond à une pause de 5 secondes.}

\begin{tabular}{|l|l|l|}
\hline Translog & TAPs & Ma traduction \\
\hline 1. [*:02.19.17] $\uplus^{\mathfrak{y}} \mathbb{y}$ & $\begin{array}{l}\text { Le retour des bonnes manières. Ja, } \\
\text { der kan man jo enten velge å } \\
\text { beholde gode manérer eller å bruke } \\
\text { etikette kanskje. (Kikker ut av } \\
\text { vinduet.) Jeg tror jeg lar tittelen stå } \\
\text { foreløpig og begynner litt lenger } \\
\text { ned. }\end{array}$ & $\begin{array}{l}\text { Le retour des bonnes manières. Je } \\
\text { peux donc choisir de garder bonnes } \\
\text { manières ou mettre étiquette. } \\
\text { (Regarde par la fenêtre.) Je crois que } \\
\text { je vais laisser le titre de côté pour } \\
\text { l'instant, et commencer un peu plus } \\
\text { bas. }\end{array}$ \\
\hline
\end{tabular}

Les 2 premières minutes et 19 secondes de la pause initiale sont consacrées à la lecture intérieure du texte original. Christine souhaite visiblement s'imprégner du contenu du texte avant d'entamer sa traduction. Comme en témoignent ses premiers commentaires, Christine se demande comment traduire bonnes manières dans le titre: elle peut soit se contenter de transposer le syntagme correspondant en norvégien: gode manerer, soit choisir un synonyme contextuel de celui-ci: etikette. Sa décision de reporter la traduction du titre à plus tard, correspond dans le premier segment Translog, au triple appui sur la touche interligne, représenté par les trois flèches.

\section{Suite des données processuelles de Christine:}

\begin{tabular}{|c|c|c|}
\hline Translog & TAPs & Ma traduction \\
\hline 2. [*:01.01.10] & $\begin{array}{l}\text { On les croyait. O.K., så det første } \\
\text { jeg må tenke på, er om jeg skal } \\
\text { beholde on, altså en litt upersonlig } \\
\text { form, eller... Skal vi se... dette er } \\
\text { en litt uvant situasjon, så jeg har } \\
\text { en liten blokkering her, men det } \\
\text { kommer seg vel etter hvert (retter } \\
\text { seg opp). Det er en veldig typisk } \\
\text { fransk tekst dette med... Den er } \\
\text { veldig tett bygd opp. Jeg tror jeg } \\
\text { begynner med etikette og ser } \\
\text { hvordan det går. } \\
\text { (Tar fram ordboka.) }\end{array}$ & $\begin{array}{l}\text { On les croyait. O.K., il me faut } \\
\text { d'abord décider si je garde on, } \\
\text { donc une forme un peu imperson- } \\
\text { nelle, ou (... Voyons voir... c'est } \\
\text { une situation peu usuelle, je } \\
\text { ressens un petit blocage ici, mais } \\
\text { ça va certainement s'arranger (se } \\
\text { redresse). C'est un texte typique- } \\
\text { ment français qui... est très } \\
\text { dense. Je crois que je vais mettre } \\
\text { etikette et voir par la suite s'il } \\
\text { convient. } \\
\text { (Cherche son dictionnaire.) }\end{array}$ \\
\hline
\end{tabular}




\begin{tabular}{|c|c|c|}
\hline 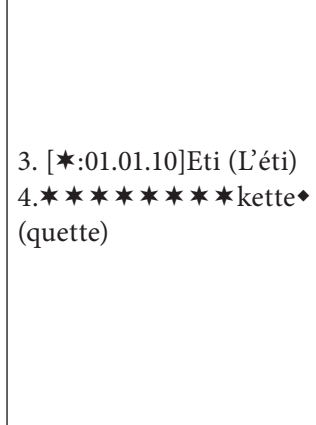 & $\begin{array}{l}\text { Skal vi se på étiquette. Det er et } \\
\text { sånt ord som jeg... Hva var den } \\
\text { boka til Randi Bratholm het, var } \\
\text { det ikke Etikette? Skikk og bruk? } \\
\text { Nei skikk og bruk, det blir litt } \\
\text { for... } \\
\text { Jeg kan jo blande litt, bruke litt } \\
\text { gode manérer og litt... } \\
\text { Ja, det går nok bedre når jeg } \\
\text { kommer inn i det, så den er grei. } \\
\text { (Skriver igjen.) } \\
\text { Etikette. }\end{array}$ & $\begin{array}{l}\text { Voyons à étiquette. C’est un mot } \\
\text { que je... Comment s'appelait donc } \\
\text { ce livre de Randi Bratholm, était- } \\
\text { ce Etikette? Skikk og bruk? Non, le } \\
\text { bon usage ça fait un peu trop... } \\
\text { Je vais mélanger un peu, tantôt } \\
\text { mettre gode manérer, et tantôt... } \\
\text { O.K., ça ira mieux quand je me } \\
\text { serai imprégnée du texte. } \\
\text { (Se remet à écrire.) } \\
\text { Étiquette. }\end{array}$ \\
\hline
\end{tabular}

Pendant sa seconde pause de plus d'une minute (voir le segment Translog 2), Christine n'a toujours rien écrit. Ressentant visiblement qu'une structure syntaxique transcodée, bien que grammaticalement acceptable en norvégien, ne convient pas, elle suggère d'écarter on en début de texte au profit d'un sujet moins vague. Elle retient etikette, l'un des deux termes proposés initialement pour la traduction de «bonnes manières» dans le titre, solution qui permet au lecteur d'entrer en plein dans le sujet du texte.

Il est intéressant de noter aussi que Christine ne s'inspire pas de son dictionnaire bilingue, qu'elle tient pourtant en mains, lorsqu'elle convoque de sa mémoire les termes etikette et skikk og bruk "bon usage». Comme en témoignent ses données TAPs, c'est une association à un livre norvégien qu'elle a lu sur le bon usage qui la met sur la voie d'une solution au problème de reformulation.

À peine Christine a-t-elle tapé les trois premières lettres du mot etikette (voir le segment Translog 3 ci-dessus), qu'elle marque une pause de 40 secondes, dans laquelle elle explique qu'elle peut alterner dans son texte entre le correspondant gode manerer et d'autres synonymes. Bien qu'elle tranche pour etikette (voir le segment Translog 4 ci-dessus où elle tape les 5 dernières lettres du mot), elle remarque que cette solution n'est pas définitive.

\section{Suite des données Translog de Christine:}

\begin{tabular}{|c|c|c|}
\hline Translog & TAPs & Ma traduction \\
\hline 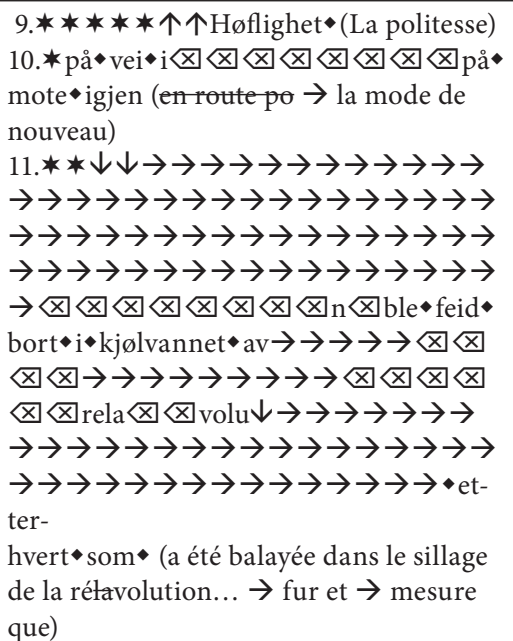 & $\begin{array}{l}\text { Høflighet, kan man si } \\
\text { det? Høflighet på vei ut. } \\
\text { Høflighet kom bort... } \\
\text { igjen... igjen må en vel } \\
\text { si... Retour. Kom bort } \\
\text { igjen. Det var det vel, ja. } \\
\text { Høflighet kom bort igjen. } \\
\text { Forsvant med. Feiet bort. } \\
\text { En kan bruke feiet bort } \\
\text { selv om ... Jeg tror } \\
\text { kanskje jeg vil satse på } \\
\text { den... I kjølvannet av } \\
\text { en... revolusjon må en } \\
\text { vel ha. Stadig etterhvert } \\
\text { som... } \\
\text { Eh (pause) } \\
\text { (gestikulerer). }\end{array}$ & $\begin{array}{l}\text { Politesse: ça se dit ça? La } \\
\text { politesse en voie de } \\
\text { disparition. La politesse } \\
\text { s'est perdue... à nouveau... } \\
\text { à nouveau... ça peut se dire. } \\
\text { Retour. S'est perdue à } \\
\text { nouveau. C'est bien ça. La } \\
\text { politesse s'est perdue à } \\
\text { nouveau. A disparu. A été } \\
\text { balayée. Je peux mettre a } \\
\text { été balayée même si... Je } \\
\text { crois que je vais opter pour } \\
\text { ça... Dans le sillage d'une... } \\
\text { révolution, ça marche. } \\
\text { Toujours au fur et à mesure } \\
\text { que... } \\
\text { Eh (pause) } \\
\text { (gesticule). }\end{array}$ \\
\hline
\end{tabular}




\begin{tabular}{|c|c|c|}
\hline 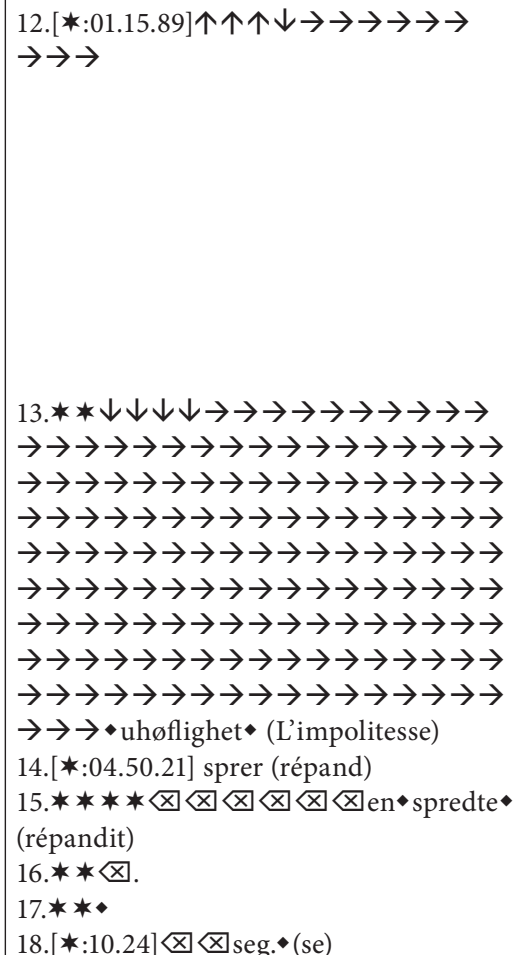 & $\begin{array}{l}\text { Høflighet. Nei, da må jeg } \\
\text { hente litt... skal vi se, } \\
\text { hente litt inspirasjon fra } \\
\text { ordboken. (Leser i den.) } \\
\text { Uhøflighet er ikke et ord } \\
\text { jeg er så veldig glad i da, } \\
\text { men... Dette burde jeg } \\
\text { vite. Hva er det de kaller } \\
\text { det, en time på skolen... } \\
\text { Skikk og bruk? (Ser ut av } \\
\text { vinduet.) (Tar seg til } \\
\text { ansiktet.) Jeg har sett det } \\
\text { så mange ganger. Hva } \\
\text { heter det? De har en time } \\
\text { i ... det er ikke skikk og } \\
\text { bruk, men noe i den } \\
\text { duren. Men det kommer } \\
\text { vel. Høflighet er det ikke } \\
\text { litt rart? } \\
\text { (Leser fra skjermen.) } \\
\text { (Skriver.) }\end{array}$ & $\begin{array}{l}\text { La politesse. Non, il me } \\
\text { faut, voyons...chercher un } \\
\text { peu d'inspiration dans mon } \\
\text { dictionnaire. (Le consulte.) } \\
\text { Impolitesse, c'est un mot qui } \\
\text { ne me plaît pas beaucoup, } \\
\text { mais... Je devrais le } \\
\text { connaître. Comment est-ce } \\
\text { qu'ils appellent ça, un cours } \\
\text { de... Bon usage? (Regarde } \\
\text { par la fenêtre.) (Se passe la } \\
\text { main sur le visage.) Je l'ai } \\
\text { vu tant de fois. Comment } \\
\text { ça se dit? Un cours de... ce } \\
\text { n'est pas le bon usage, mais } \\
\text { quelque chose dans ce } \\
\text { genre. Ça va sans doute me } \\
\text { revenir. Politesse, n'est-ce } \\
\text { pas un peu bizarre? } \\
\text { (Se relit.) } \\
\text { (Écrit.) }\end{array}$ \\
\hline
\end{tabular}

Christine n'a pas même fini de traduire la première phrase qu'elle remonte au titre comme en témoignent les deux flèches $\uparrow \uparrow$ dans le segment Translog 9 ci-dessus, à la suite de quoi elle inscrit Høflighet på mote igjen «La politesse à la mode de nouveau». Puis suit un va-et-vient entre le corps du texte et le titre. Elle:

(i) revient à sa traduction de la première phrase (voir le segment Translog 11),

(ii) remonte ensuite au titre après une longue pause d'1 minute et 15 secondes (voir le segment Translog 12), sans pourtant rien y changer,

(iii) retourne au corps du texte où elle achève de traduire la première phrase (voir les segments Translog 13 à 18) en optant pour uhøfligheten spredte seg "L'impolitesse se répandit.»

Ses données TAPs révèlent que la solution retenue ne lui plaît guère. Sans doute estce la personnification de høflighet en norvégien qui la gêne. Par voie d'association d'idées, elle tente de convoquer de sa mémoire le nom donné aux cours de savoir-vivre à l'école secondaire en Norvège. Mais comme le terme lui échappe, elle abandonne cette voie d'exploration dans l'espoir qu'il puisse lui revenir à l'esprit plus tard, et se tourne vers son dictionnaire pour s'en inspirer. Or, celui-ci ne s'avérant guère utile, elle décide à contre-cœur de traduire «incivilités» par l'antonyme de politesse: uhøflighet. Ce choix, qui semble découler d'un rapport établi avec le titre, illustre comment deux segments textuels différents, les bonnes manières et les incivilités dans deux propositions différentes sont reliés entre eux par un rapprochement antonymique contextuel. Est-ce la traduction du terme «incivilités» par uhøflighet qui l'a amenée à faire ce choix, ou est-ce la traduction de «bonnes manières » par høflighet qui l'a inspirée à retenir son antonyme uhøflighet «impolitesse » pour traduire «inci- 
vilités»? La question reste ouverte du fait que ses données processuelles ne permettent pas de trancher. N'empêche que ce rapport antonymique est si fort que malgré ses nombreux efforts pour trouver une autre solution - elle avouera d'ailleurs que sa prose n'est pas brillante à cet égard dans ses commentaires TAPs pendant la très longue pause de 4 minutes et 50 secondes (le segment Translog 14 ci-dessus) consacrés pour l'essentiel à la traduction de l'idée contenue dans « recrudescence»-, c'est bien celui qu'elle retiendra, sans grand enthousiasme, dans sa traduction définitive.

Nous constatons que la comparaison interlinguistique n'intervient pas dans la recherche d'une formulation appropriée en norvégien. Christine consulte, certes, l'entrée høflighet dans son dictionnaire, mais comme celui-ci ne lui apporte pas le terme qu'elle a sur le bout des lèvres, il ne lui est d'aucune utilité. Sa démarche pour se sortir du blocage comprend quatre temps:

(i) Elle mobilise son souvenir d'un livre sur le bon usage en Norvège. Or, comme le titre significatif du livre lui échappe,

(ii) elle tâche de se remémorer le nom donné aux cours de savoir-vivre à l'école secondaire norvégienne. Cette remémoration n'ayant pas abouti,

(iii) elle se rabat sur son dictionnaire bilingue dans l'espoir qu'il puisse la mettre sur la voie du terme sollicité. Cette recherche étant restée vaine,

(iv) elle établit un rapport antonymique entre deux termes du texte.

Les activités déployées dans (i) et (ii) découlent d'une représentation mentale, fruit d'une activation du bagage cognitif pertinent de Christine. Ce n'est que lorsque ce type d'activité conceptuelle échoue, que Christine a recours à son dictionnaire (iii). Celuici ne donnant pas le résultat escompté, elle effectue un rapport antonymique (iv).

\subsection{Données processuelles d'Anne relatives à l'énoncé 2): Mais elles restent aussi, plus sournoisement, un mode de tri social où chacun des astérisques qui n'est pas mis entre crochets correspond à une pause d'une seconde.}

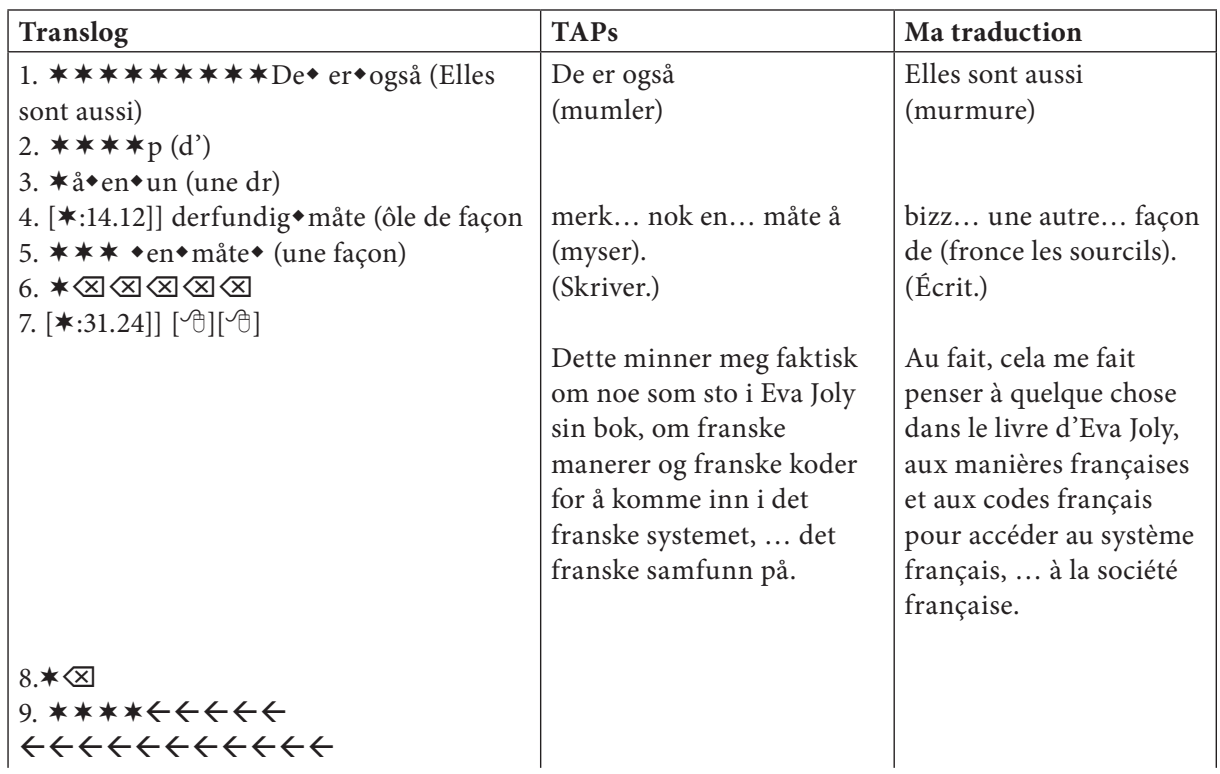




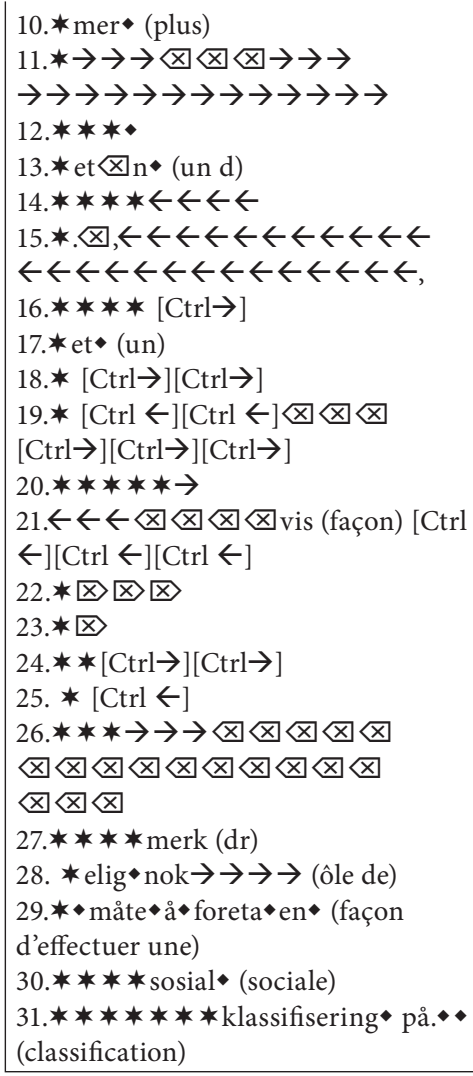

Seul sera examiné ici le syntagme métonymique «mode de tri social». La pause de 31,24 secondes dans le segment 7 de Translog ci-dessus est consacrée aux réflexions d'Anne sur un passage du dernier livre d'Éva Joly portant sur les bonnes manières en France. Comme nous l'indiquent ses commentaires verbaux, c'est par le biais de l'association faite à ce livre qu'elle suggère de traduire «mode de tri social » par foreta en sosial klassifiering på: "façon d'effectuer une classification sociale». Nous remarquons qu'Anne n'a éprouvé aucune difficulté à comprendre l'énoncé original. Ses efforts portent sur la meilleure façon de traduire le syntagme «mode de tri social» en norvégien. Ses données TAPs nous permettent de noter que sa reformulation repose sur l'adjonction de ses compléments notionnels au sémantisme du texte. Comme dans le cas précédent, la solution d'Anne découle, non pas d'une comparaison interlinguistique, mais d'une représentation mentale: son souvenir du livre, fruit de l'activation de son bagage cognitif pertinent. 


\subsection{Les données processuelles de Brigitte relatives à l'énoncé 3): S'il est désormais bien vu de laisser au placard cravate et costume trois pièces le vendredi [...] où chacun des astérisques correspond à une pause de 5 secondes.}

\begin{tabular}{|c|c|c|}
\hline Translog & TAPs & Ma traduction \\
\hline 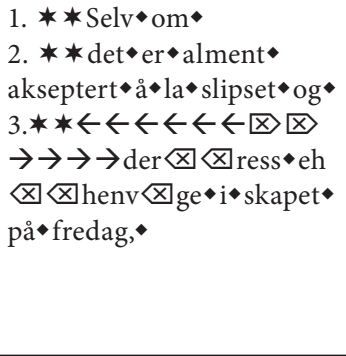 & $\begin{array}{l}\text { Costume trois pièces... (Klør } \\
\text { seg i armen.) Det er jo da, } \\
\text { ikke sant, dress med vest, } \\
\text { men jeg sier dress, jeg. Jeg } \\
\text { dropper det (mumler) med... } \\
\text { trois pièces (klyper seg i } \\
\text { nesen). }\end{array}$ & $\begin{array}{l}\text { Costume trois pièces... (Se gratte } \\
\text { le bras.) Il s'agit bien d'un } \\
\text { costume avec un gilet, mais je } \\
\text { vais simplement mettre } \\
\text { costume. Je laisse tomber } \\
\text { (inaudible) ce ... trois pièces (se } \\
\text { pince le nez). }\end{array}$ \\
\hline
\end{tabular}

Brigitte sait parfaitement ce qu'est un «costume trois pièces», puisqu'elle en donne une explication: "un costume avec un gilet». Or, dans sa traduction norvégienne, seul figure le terme dress («costume»). Bien que ses données TAPs au sujet de la raison pour laquelle elle a choisi de ne pas traduire «gilet» soient inaudibles, ses données comportementales, à savoir les gestes subconscients qui précèdent et succèdent sa solution, tels le fait de se gratter le bras, puis de se pincer le nez, signalent un effort cognitif sous-jacent à sa solution. Le gilet étant aujourd'hui associé au smoking porté dans les grandes occasions en Norvège, il est tentant de voir dans l'omission de Brigitte un choix délibéré. Le terme dress qui ne recouvre que le pantalon et la veste, désigne parfaitement l'idée d'une tenue vestimentaire formelle au bureau. C'est bien l'idée véhiculée par les mots que retient et reformule Brigitte, et non pas les mots de l'original. Si ces derniers avaient été reportés tels quels, ils auraient détourné l'attention du lecteur norvégien sur une situation autre que celle de la tenue vestimentaire usuelle au bureau évoquée par l'auteur du texte original et, le cas échéant, contribué à une déviation du sens. Soucieuse de transmettre à ses lecteurs norvégiens un contenu dénotatif équivalent à l'original français, seuls les sèmes actualisés en fonction du message à transmettre ont donc été restitués dans sa traduction norvégienne. Brigitte a recouru en norvégien au choix d'un vocable moins riche en sèmes que celui de la langue de départ, lequel transmet pourtant parfaitement le même sens ${ }^{1}$. Une partie pour le tout - différente certes de la partie retenue par l'auteur du texte original français - mais renvoyant au même tout de façon parfaitement idiomatique en norvégien.

\section{Conclusion}

La traduction étant par définition un processus complexe de résolution de problèmes, mieux comprendre la démarche cognitive de l'expert-traducteur face à un blocage de reformulation, ne peut que faire progresser les études en traduction. Grâce aux données processuelles in vivo recueillies par les commentaires TAPs et les activités d'écriture enregistrées par le logiciel Translog des sujets traduisants, il est désormais possible de: 
- voir à quel endroit un blocage se produit;

- décrire la nature du blocage;

- déceler le type d'activité entreprise pour sortir du blocage, à savoir la démarche cognitive du sujet traduisant;

- dégager les principes de traduction sous-jacents.

L'analyse des données processuelles a permis de montrer que les trois professionnelles de la traduction ont relevé le défi du blocage ressenti au niveau de la reformulation en adjoignant des compléments notionnels au sémantisme du texte original. C'est en puisant dans leur savoir encyclopédique et dans les coutumes de la culture norvégienne qu'elles ont pu débloquer la situation et proposer une solution acceptable dans la langue d'arrivée.

Le démontage de la démarche cognitive du traducteur professionnel fondé sur l'analyse in vivo fournit à la traductologie des données empiriques précieuses sur le «comment» de la formulation des idées dans la langue d'arrivée, à partir desquelles un certain nombre d'hypothèses sur la traduction, restées non validées expérimentalement, peuvent être vérifiées. Nous avons vu ici que la comparaison interlinguistique n'est pas le procédé de reformulation auquel ont eu recours les trois experts-traducteurs, dès lors qu'une visualisation du sens des énoncés métaphorique et/ou métonymique a eu lieu. Cette visualisation, ou représentation mentale - dont les retombées linguistiques ne se recoupent pas forcément avec l'expression linguistique originale - est le résultat d'une adjonction, au sémantisme du texte original, de compléments notionnels.

S'il est vrai, dans un cadre unilingue, qu'un seul et même individu est capable de s'imaginer une scène au moyen de représentations conceptuelles différentes, pourquoi n'en irait-il pas de même du traducteur? Bien ancré dans ses deux cultures et deux langues, il est parfaitement à même de recouvrir l'idée conceptualisée du revêtement linguistique requis par la situation contextuelle dans la langue d'arrivée. Ainsi le point de vue de Langacker selon lequel deux expressions linguistiques différentes peuvent désigner la même situation objective, s'applique tout aussi bien à la traduction qu'à la communication unilingue:

Two linguistic expressions can therefore designate the same objective situation yet differ substantially in their semantic import because they structure it through different images. (Langacker 1991: 35)

\section{NOTES}

1. Les deux autres experts-traducteurs ont également omis de traduire «veste» en norvégien. Christine a choisi la même solution que Brigitte, alors qu’Anne a réduit le syntagme métonymique «cravate et costume trois pièces» au seul terme: dress (= costume) en norvégien. Ce choix délibéré de sa part est précédé d'un commentaire TAPs sur l'inutilité de donner des précisions superflues. En d'autres termes, Anne transmet l'idée de la tenue vestimentaire formelle au bureau au moyen du trait qui la caractérise en norvégien.

\section{RÉFÉRENCES}

Barcelona, A. (ed.) (2000): Metaphor and Metonymy at the Crossroads - A Cognitive Perspective, Berlin/New York, Mouton de Gruyter.

Barcelona, A. (2003): "The case for a metonymic basis of pragmatic inferencing: Evidence from jokes and funny anecdotes”, in Panther, K.-U. and L. Thornburg (eds.), Metonymy and Pragmatic Inferencing, Amsterdam/ Philadelphia, John Benjamins. 
Croft, W. (2003): "The role of domains in the interpretation of metaphors and metonymies", in Dirven, R. and R. Pörings (eds.), Metaphor and Metonymy in Comparison and Contrast, Berlin/New York, Mouton de Gruyter, pp. 161-205.

Delisle, J. (1984): L'analyse du discours comme méthode de traduction, Ottawa, Presses de l'Université d'Ottawa.

Ericsson, K. and H. Simon (1993): Protocol Analysis: Verbal Reports as Data, Cambridge, The MIT Press.

Gerloff, P. (1986): “Second language learners' reports on the interpretive process: Talk aloud protocols of translation", in House, J. and S. BLUM-KulKa (eds), Interlingual and Intercultural Communication, Tübingen, Gunter Narr, pp. 243-262.

Gibis, R.W. (1994): The Poetics of Mind, Cambridge, Cambridge University Press.

Goossens, L. (2003): "Metaphtonymy: the interaction of metaphor and metonymy in expressions for linguistic action", in Dirven, R. and R. Pörings (eds), Metaphor and Metonymy in Comparison and Contrast, Berlin/New York, Mouton de Gruyter, pp. 349-377.

Jakobsen, L. A. (1999): "Logging time delay in translation", in Hansen, G. (ed.), Probing the Process in Translation: Methods and Results, Copenhagen, Copenhagen Business School, pp. 73-101.

Jakobsen, L. A. (2000): "Understanding the Process of Translation: The Contribution of TimeDelay Studies", in Englund Dimitrova, B. (ed.), Översättning och tolkning, ASLA:s skriftserie 12, pp. 155-172.

Jensen, A. (2000): The Effects of Time on Cognitive Processes and Strategies in Translation, Ph.D. thesis, Copenhagen Business School.

JäÄskelaïnen, R. (1987): What happens in a Translation Process: Think-aloud Protocols of Translation, Joensuu, University of Joensuu, Savonlinna School of Translation Studies, pp. 87-98.

Jä̈̈skeläInen, R. (2000): Tapping the Process - An Explorative Study of the Cognitive and Affective Factors Involved in Translating, Joensuu, University of Joensuu, Savonlinna School of Translation Studies.

KRINGS, H. P. (1986): “Translation problems and translation strategies of advanced German learners of French (L2)", in House, J. and S. BLum-KulKa (eds), Interlingual and intercultural communication, Tübingen, Gunter Narr, pp. 263-275.

Lakoff, G. and M. Turner (1989): More than Cool Reason. A Field to Poetic Metaphor, Chicago/London, The University of Chicago Press.

LakofF, G. and M. Johnson (1980): Metaphors We Live By, Chicago/London, The University of Chicago Press.

LAKOFF, G. (1996) : «Les universaux de la pensée métaphorique: la question de la variation dans l'expression linguistique», actes du colloque Diversité des langues et représentations cognitives, Paris, <http://www.ltm.ens.fr/chercheurs/lassegue/traductions1.\%20trad.ling.html>.

LANGACKer, R. (1991): Concept, Image, and Symbol - The Cognitive Basis of Grammar, Berlin/ New York, Mouton de Gruyter.

Lederer, M. (1981): La traduction simultanée - fondements théoriques, Paris, Lettres Modernes Minard.

Lederer, M. (1994): La traduction aujourd'hui - Le modèle interprétatif, Paris, Hachette.

LÖrscher, W. (1986): "Linguistic aspects of translation processes: Towards an analysis of translation performance", in House, J. and S. Blum-Kulka (eds), Interlingual and intercultural communication, Tübingen, Gunter Narr, pp. 277-292.

Panther, K.-U. and G. Radden (eds.) (1999): Metonymy in Language and Thought, Amsterdam/Philadelphia, John Benjamins.

Panther, K.-U. and L. Thornburg (eds.) (2003): Metonymy and Pragmatic Inferencing, Amsterdam/Philadelphia, John Benjamins.

Panther, K.-U. and L. Thornburg (2004): The Role of conceptual Metonymy in Meaning Construction, <http://www.metaphorik.de/06/pantherthornburg.htm>. 
Radden, G. (2003): “How metonymic are metaphors?", in Dirven, R. and R. Pörings (eds), Metaphor and Metonymy in Comparison and Contrast, Berlin/New York, Mouton de Gruyter, pp. 75-111.

Rydning, A. F. (2002) : «Concept métaphorique et expression métaphorique dans une perspective cognitiviste», in Dørum, H. (ed.), Romansk Forum 16-2, pp. 723-733.

Rydning, A. F. (2004) : «Le défi du procédé synecdoquien en traduction», Meta 49-4, pp. 856875.

Rydning, A. F. (2005): «Étude de l'effort cognitif du traducteur lié à la reformulation de métaphores», in IsRaëL, F. et M. LEDERER (eds), La théorie interprétative de la traduction. Convergences, mises en perspective, Paris, Lettres modernes Minard, pp. 265-295.

SCHILPERoord, J. (1996): It's about Time: Temporal Aspects of Cognitive Processes in Text Production, Amsterdam, Rodopi.

SÉGuinot, C. (1989): “The translation process: An experimental study”, in SÉGuinot, C. (ed.), The Translation Process, Toronto, H.G. Publications, pp. 21-53.

TAYloR, J. (2003): "Category extension by metonymy and metaphor", in DiRven, R. and R. Pörings (eds.), Metaphor and Metonymy in Comparison and Contrast, Berlin/New York, Mouton de Gruyter, pp. 323-347.

Thornburg, L. and K.-U. Panther (1997): "Speech act metonymies", in Liebert, W-A., Redeker, G. and L. Waugh (eds.), Discourse and Perspective in Cognitive Linguistics, Amsterdam/Philadelphia, John Benjamins, pp. 205-219.

Tirkkonen-Condit, S. (1989): "Professional vs Non-professional translation: A think-aloud protocol study”, in SÉGuinot, C. (ed.) The Translation Process, Toronto, H.G. Publications, pp. 73-85.

Tirkkonen-Condit, S. (2002): "Process Research: State of the Art and Where to Go Next?", Across Languages and Cultures 3-1, Akadémiai Kiadó, pp. 5-19. 